\title{
Artificial Knee Joints Actuators with Energy Recovery Capabilities: A Comparison of Performance
}

\author{
Roberta Alò, Francesco Bottiglione, and Giacomo Mantriota \\ Dipartimento di Meccanica, Matematica e Management, Politecnico di Bari, Viale Japigia 182, 70126 Bari, Italy \\ Correspondence should be addressed to Roberta Alò; roberta.alo@poliba.it
}

Received 13 October 2015; Accepted 15 February 2016

Academic Editor: Shahram Payandeh

Copyright (C) 2016 Roberta Alò et al. This is an open access article distributed under the Creative Commons Attribution License, which permits unrestricted use, distribution, and reproduction in any medium, provided the original work is properly cited.

\begin{abstract}
The human knee absorbs more energy than it expends in level ground walking. For this reason it would be useful if the actuation system of a wearable robot for lower limbs was able to recover energy thus improving portability. Presently, we recognize three promising technologies with energy recovery capabilities already available in the literature: the Series Elastic Actuator (SEA), the Clutchable Series Elastic Actuator (C-SEA), and the flywheel Infinitely Variable Transmission (F-IVT) actuator. In this paper, a simulation model based comparison of the performance of these actuators is presented. The focus is on two performance indexes: the energy consumed by the electric motor per gait and the peak torque/power requested to the electric motor. Both quantities are related to the portability of the device: the former affects the size of the batteries for a given desired range; the latter affects the size and the weight of the electric motor. The results show that, besides some well-explained limitations of the presented methodology, the C-SEA is the most energy efficient whereas the F-IVT allows cutting down the motor torque/peak power strongly. The analysis also leads to defining how it is possible to improve the F-IVT to achieve a reduction of the energy consumption.
\end{abstract}

\section{Introduction}

In the human normal gait cycle, the knee joint undergoes phases of positive and negative work. Therefore an optimally designed wearable device for lower limbs (orthoses, exoskeletons, and prostheses) should be able to store energy during the phases of negative work and to permit reusing it when required. Indeed, one of the greatest limits to the development of powered orthoses, exoskeletons, and prostheses deals with their portability that is often limited by the lack of actuators and energy sources enabling the development of devices with both reasonable weight and an acceptable range of locomotion [1]. The energy recovery during normal operation is a smart strategy to reduce the net energy consumption. It could extend the portability of wearable devices enlarging their operating range and reducing the power requested to the motor.

Most of the wearable devices for lower limbs rely on battery-powered electric motors [2]. Reversibility of the electric drives permits in principle the regeneration of electric energy to be stored in a battery pack. However, in the context of wearable robots for lower limbs, the charging/discharging cycles of the battery occur under variable tension/current conditions and their frequency is relatively high, resulting in a very large number of cycles during the lifetime of the device, which adversely affects the performance and the life of the battery $[3,4]$. The relatively low efficiency of the mechanical to electrical energy conversion and of the battery charge/discharge process and the high frequency of the gait cycle are the reasons why the electric energy recovery has not usually been considered in this kind of application $[5,6]$ up to very recent times [7].

Great efforts are focused on developing mechanical energy storage devices, in which the lack of any energy conversion would result in a greater efficacy. SEAs (Series Elastic Actuators) include an elastic element between the motor and the load which stores energy and delivers peak power values greater than the maximum power of the motor, and it offers greater shock tolerance, lower reflected inertia, and more accurate and stable force control [8-11]. In SEAs, the linear 
torque-angle relationship of the early stance knee flexionextension phase of human walking can be rendered only by a spring whose stiffness is equal to the slope of the torqueangle curve. VSAs (Variable Stiffness Actuators) which adapt their stiffness to match the torque-angle curve also during the swing-phase [12] are claimed to achieve greater energy saving and peak power decrease compared to SEAs. Furthermore VSAs are able to adjust the optimal value of their stiffness according to the load conditions, permitting obtaining greater benefits at more than one specific walking gait.

In SEAs, when only the spring powers the knee joint, the motor must provide only reactionary torque at negligible motor speed, thus working inefficiently [7]. This consideration motivated the improvement of the design of SEAs into the development of the C-SEA (Clutchable Series Elastic Actuator), where a clutch is activated or deactivated according to the phase of the gait cycle. The C-SEA is explained in detail in [7], where its performance is evaluated in powering knee prostheses. It was proved that the C-SEA reduces the net electric energy consumption also thanks to the electric energy recovery. However, a limitation of such device is that when the clutch is activated the motor is not able to provide additional torque; thus the stiffness of the spring limits the operation of the C-SEA mechanism to those activities whose torque demand is into the bandwidth of the device, which is defined by the stiffness value.

Another way to store mechanical energy involves kinetic energy storage in a high speed rotating mass (flywheel). The flywheel energy storage has been recently reconsidered in the automobile field under the denomination of mechanical KERS (Kinetic Energy Recovery System). It is one possible short-term solution to achieve an improvement of the fuel economy performance of automobiles. In mechanical KERS, the energy is moved from the load (vehicle) to the flywheel under negative work phases and from the flywheel to the load otherwise [13] with no energy transformation. Inspired by KERS, the F-IVT (flywheel-IVT) actuator has been presented by the authors [14] as a device leading to potential benefits when employed in powering knee bionic joints. The F-IVT includes a brushless DC motor, a flywheel, an IVT (Infinitely Variable Transmission), and HD (harmonic drive) gear. The flywheel acts as a kinetic energy storage device; energy is moved between the motor and the joint thanks to a proper change of the speed ratio of the IVT. The IVT, whose ratio changes between positive and negative values, also permits a nearly constant motor speed, while the desired joint speed is, in principle, always matched.

In [14] the performance of the F-IVT was predicted in one specific walking condition, for which it was optimally designed. The authors proved that the F-IVT architecture permits undersizing the electric motor, which in F-IVT delivers an almost constant power value roughly equal to the mean power requested and by far less than the peak power. Moreover, the electric motor can be sized for working at a nearly fixed point at the greatest efficiency values, with a resulting potential saving of electric energy consumption. The motor undersizing would be a great advantage in this kind of application because it facilitates the portability of the device, also enlarging its operating range.
However, the results described in [14] give a limited insight into the F-IVT potential because the performance is therein estimated in the nominal working condition, that is, the one taken as a reference to optimally design the FIVT components. Indeed, the portable lower limb wearable robots are required to support the wearer in his daily activity; thus their actuators do not usually work under the nominal condition.

The aim of this analysis is to further investigate the FIVT by predicting its performance when it does not work under the condition for which it was optimally designed, like different walking speeds and stairs climbing. The energy and power requirements of the F-IVT were estimated through a model based approach and compared with those of two very efficient actuators already used in biomechatronics devices, the SEA and the C-SEA, in order to evaluate if it could be a competitive actuator for artificial knee joints of wearable robots.

\section{Materials and Methods}

This study compares through a model based approach three different actuators for powering artificial knee joints: the FIVT, a novel actuator presented very recently in [14], and two other high performance actuators, the SEA and the C-SEA, which were largely investigated in previous works $[3,7,15,16]$.

The actuators F-IVT, SEA, and C-SEA were compared in different working conditions: level ground walking at different speeds $(0.5,1.1,1.6$, and $2.1 \mathrm{~m} / \mathrm{s})$ and stairs climbing at different inclinations $\left(24^{\circ}, 30^{\circ}\right.$, and $\left.42^{\circ}\right)$. These conditions often recur in daily life. Indeed, it would be desirable for any actuator of artificial human joints to support the subject in his daily activity, requiring both a large operating range and the portability of the device.

Two main performance indexes have been taken into consideration for comparison: the energy consumption (per gait) and the peak of electric power, both calculated at the motor electrodes. These are significant quantities for the development of lightweight and portable wearable robots because they affect the size and the weight of the actuator, as well as the operating range of the device. The energetic and power requirements of all the actuators were calculated through an inverse dynamic approach, that is to say, starting from the load characteristics (knee angle and torque versus time) given as input data to the model provided in Sections 2.1-2.3. Gait cycles data were taken from $[17,18]$ (Figure 1).

The following sections provide details on the working principle of all the actuators, as well as on their mathematical modeling and the sizing procedure. Both SEA and C-SEA were modeled and sized as already done in the literature.

2.1. The Flywheel-IVT (F-IVT) Actuator. The F-IVT is an innovative actuator with energy recovery capabilities to power artificial knee joints [14]. It is made up of the following devices: a brushless DC motor, a flywheel, an IVT, and a HD gear (Figure 2). The brushless DC motor and the HD gear are often employed in biomechatronics: the first one because of its great efficiency with respect to other electric drives [19], the second one because of its large torque capacity, high gear 


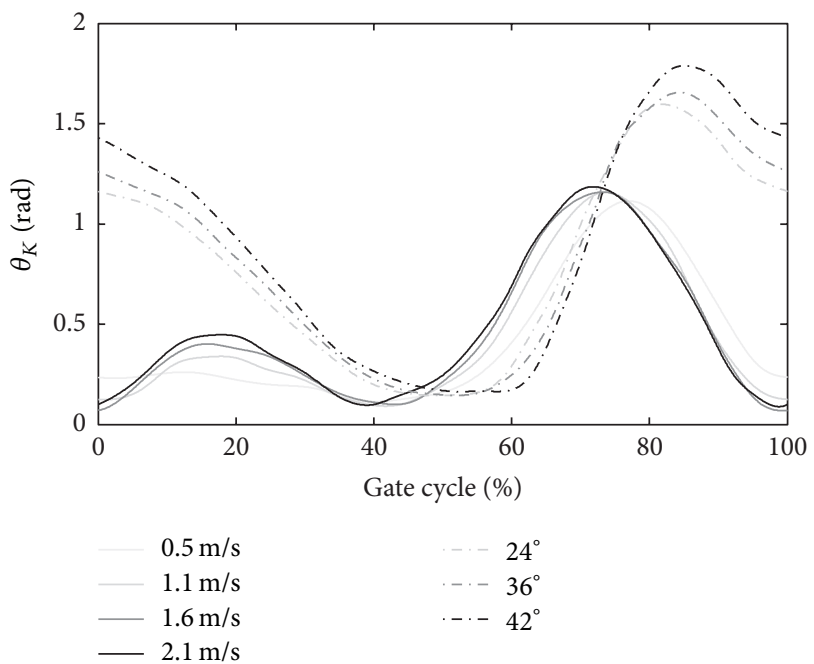

(a)
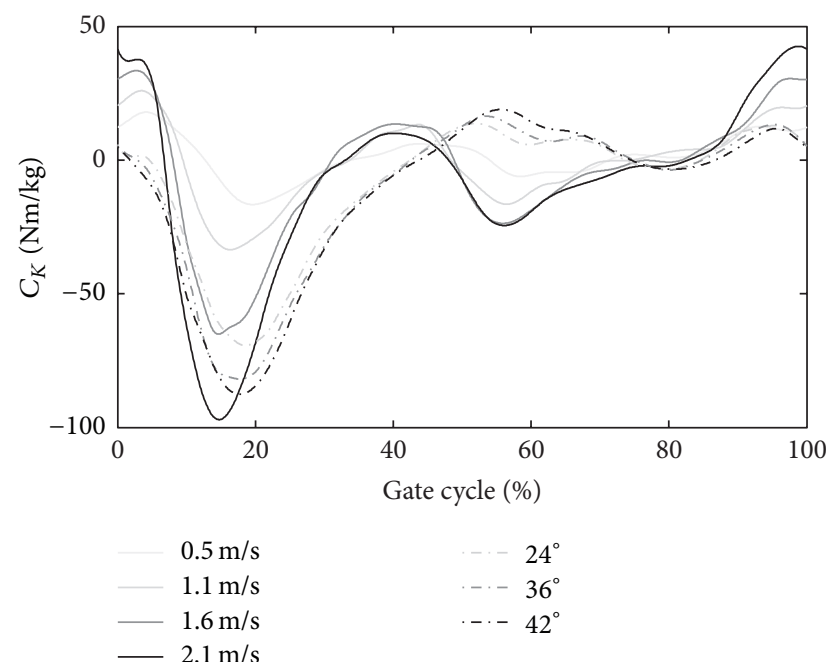

(b)

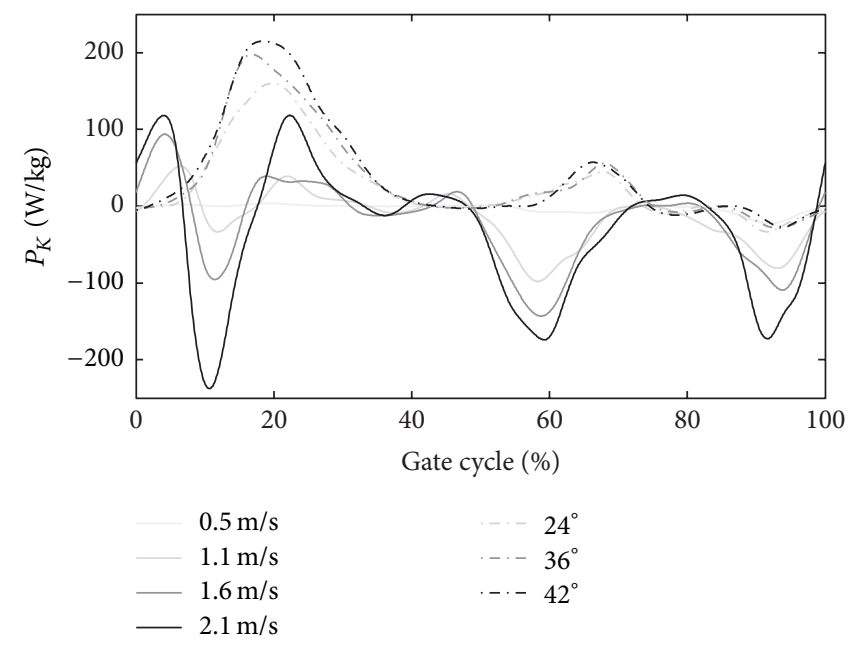

(c)

FIGURE 1: Gait cycle analysis data of the knee joint (angle, torque, and power) in level ground walking at different speeds and in stairs climbing at different inclinations. Data were adapted from $[17,18]$.

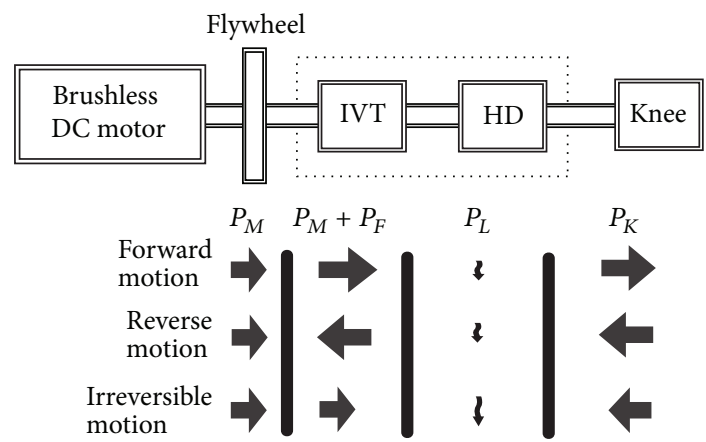

FIGURE 2: Fluxes of power in the F-IVT under forward, reverse, and irreversible motion conditions. The arrows indicate the power flows direction in each of them $\left(P_{M}\right.$ motor power, $P_{F}$ rate of change of the kinetic energy of the flywheel, $P_{L}$ power losses in the transmission devices, and $P_{K}$ knee power). ratios $\left(\tau_{\mathrm{HD}}\right)$, and small size [19]. The flywheel and the IVT are introduced for the first time in an actuation system of artificial leg joints with the aim of recovering energy and reducing the irregularity of the motor working point.

As in a mechanical KERS (Kinetic Energy Recovery System) [13], in the F-IVT the flywheel operates as a kinetic energy storage device in which energy is stored when the power demand of the knee is negative (reverse motion) and it is released otherwise (forward motion), as schematically depicted in Figure 2. Energy is moved between the motor and the joint through a proper change of the speed ratio of the Continuously Variable Transmission. More specifically, an IVT is required because of the alternate motion of the knee joint. The speed ratio of the IVT $\left(\tau_{\mathrm{IVT}}\right)$ is changed continuously between positive and negative values $\left(\tau_{\text {IVT }}^{\mathrm{min}} \leq \tau_{\text {IVT }} \leq\right.$ $\left.\tau_{\text {IVT }}^{\max }\right)$ permitting achieving the desired joint speed $\left(\omega_{K}\right)$ while 
the motor speed is nearly constant $\left(\omega_{M}=\omega_{K} /\left(\tau_{\mathrm{IVT}} \tau_{\mathrm{HD}}\right)\right)$ even when the output speed is zero thanks to the neutral gear condition of the IVT. An IVT with shunted CVT architecture was considered in the F-IVT actuator, because mathematical models of its efficiency are well established in the literature [20-22]. It is furthermore assumed that the speed ratio of the IVT is always perfectly regulated in order to be equal to the required value of $\tau_{\text {IVT }}$ at each instant of the gait cycle.

The operation of the F-IVT is such that the motor works at nearly fixed point with high efficiency and with a resulting possibility of undersizing the motor. Indeed, in the F-IVT the motor must provide an almost constant power, while the flywheel provides the variations of the requested power at the knee joint, permitting satisfying the following instantaneous power balance equation:

$$
P_{K}=P_{M}+P_{F}+P_{L}
$$

where $P_{K}$ is the knee power, $P_{M}$ is the motor power, $P_{F}$ $\left(P_{F}=-J_{F} \dot{\omega}_{M} \omega_{M}\right)$ is the rate of change of the kinetic energy of the flywheel of inertia $J_{F}$, which rotates with the same motor angular speed $\left(\omega_{M}\right)$ and acceleration $\left(\dot{\omega}_{M}\right)$, and $P_{L}$ is the power lost in the transmission devices.

With the motor speed being nearly constant $\omega_{M} \approx \bar{\omega}_{M}$ $\left(\bar{\omega}_{M}\right.$ is the average velocity of the motor), in order to achieve an almost constant power delivery, the motor is supposed to be torque controlled with constant torque $C_{M}$ given by

$$
C_{M}=\frac{1}{T \bar{\omega}_{M}} \int_{0}^{T}\left(P_{K}(t)-P_{L}(t)\right) d t
$$

where $T$ is the period of the gait cycle, $\bar{\omega}_{M}$ is the mean value of the motor angular speed, and $\int_{0}^{T}\left(P_{K}(t)-P_{L}(t)\right) d t$ is the total amount of energy that the motor must provide to fulfill the knee power requirement and to overcome the power lost in the transmission devices.

Detailed efficiency models were adopted for the IVT and for the $\mathrm{HD}$ to realistically estimate the power losses. $P_{L}$ (see (1)) can be written as a function of the instant values of the efficiency of the IVT $\left(\eta_{\text {IVT }}\right)$ and the HD gear $\left(\eta_{\mathrm{HD}}\right)$ in forward $\left(P_{L}=-\left(1-\eta_{\mathrm{IVT}} \eta_{\mathrm{HD}}\right)\left(P_{M}+P_{F}\right)\right)$ and reverse $\left(P_{L}=-(1-\right.$ $\left.\left.\eta_{\mathrm{IVT}} \eta_{\mathrm{HD}}\right) P_{K}\right)$ motion. The adopted efficiency models also consider the nonreversible motion condition, which implies that $P_{M}, P_{F}$, and $P_{K}$ are all delivered to the transmission and then dissipated as heat losses (Figure 2). This situation can involve the HD, the IVT, or both simultaneously, depending on the actual values of speed and torque. In particular, we adopted the model given in [20] (described in detail in Appendix A) for predicting $\eta_{\mathrm{IVT}}$ (Figure $3(\mathrm{a})$ ), and we always referred to the guidelines of the data sheet of $\mathrm{HD}$ gears given by one manufacturer (AG Harmonic Drive) to estimate $\eta_{\mathrm{HD}}$ (Figure 3(b)) in this paper.

To determine the actual working conditions of the actuator, the equations given above are solved through an iterative routine (Figure 4) aimed at calculating the mechanical motor quantities $\omega_{M}, \dot{\omega}_{M}, C_{M}$. The iterative calculation is necessary to find the actual value of the motor speed, which is not known a priori. Starting from a guess value of constant motor speed $\omega_{M}^{(0)}$, the corresponding values of $C_{M}$ (see (2)) and then of $\dot{\omega}_{M}$ (from (1)) are calculated. $\dot{\omega}_{M}$ is then integrated through a numerical method (Newton-Raphson method) to find $\omega_{M}^{(1)}$. At integration step $j$, an error is defined as $\operatorname{ERR}_{j}=(1 / T) \int_{0}^{T}\left(\omega_{M}^{(j+1)}-\omega_{M}^{(j)}\right)^{2} d t$. The iteration stops when $\mathrm{ERR}_{j} \leq \mathrm{ERR}_{\text {max }}$, where $\mathrm{ERR}_{\text {max }}$ is the error tolerance, and it is requiring also that the constraint on the IVT ratio is satisfied $\left(\tau_{\text {IVT }}^{\min } \leq \tau_{\text {IVT }} \leq \tau_{\text {IVT }}^{\max }\right)$.

After the mechanical motor requirements are defined, the electric power requested to the motor $\left(P_{\mathrm{EL}}=v_{M} i_{M}\right)$ is calculated as a function of the motor parameters (motor torque constant $k_{t}$, damping friction coefficient $v$, Coulomb friction torque $C_{\mathrm{fr}}$, motor resistance $R$, back EMF constant $k_{\text {emf }}$, and motor inertia $J_{M}$ ) and of the calculated requirements (motor torque $C_{M}$, angular motor speed $\omega_{M}$, and angular motor acceleration $\left.\dot{\omega}_{M}\right)$ :

$$
\begin{aligned}
& i_{M}=\frac{C_{M}}{k_{t}}+\frac{J_{M} \dot{\omega}_{M}}{k_{t}}+\frac{\nu \omega_{M}}{k_{t}}+\frac{\operatorname{sign}\left(\omega_{M}\right) C_{\mathrm{fr}}}{k_{t}}, \\
& v_{M}=R i_{M}+k_{\mathrm{emf}} \omega_{M},
\end{aligned}
$$

where $i_{M}$ and $v_{M}$ are, respectively, the electric motor current and voltage.

Finally, the electric energy to be supplied is calculated as the time integral of the motor electric power $P_{\mathrm{EL}}=v_{M} i_{M}$ in the gait cycle; thus the energy consumption of all the electronic modules was neglected. According to [23], in the reverse operation it may happen that the electric motor is not able to generate electricity. In those cases, $P_{\mathrm{EL}}=0$. Consider

$$
E_{\mathrm{EL}}=\int_{0}^{T} P_{\mathrm{EL}} d t
$$

The mathematical model of the F-IVT given above (see (1)(4)) is implemented to estimate the performance of the F-IVT. In this work the F-IVT sizing was carried on in such a way that it is capable of powering the knee joint under different locomotion regimes, namely, the level ground walking at different speeds and the stairs climbing at different inclinations. In particular, the size of the HD gear and that of the motor were determined from the maximum power requested considering all possible walking regimes, whereas the most frequent one (level ground walking at $1.1 \mathrm{~m} / \mathrm{s}$ ) was used to optimally design the F-IVT in order to make it work very efficiently most of the time. More details about the criterion followed to size each component of the F-IVT, the nominal values of which are listed in Table 1, can be found in Appendix B.

2.2. Series Elastic Actuator (SEA). SEAs have been extensively employed in wearable devices for lower limbs. In SEAs a spring is put in series between the motor and the artificial powered joint (Figure 5(a)). The elastic element gives advantages to the actuation system such as increasing of shock tolerance and limited high frequency actuator impedance and energy storage [8-10]. Since the force/displacement characteristic of the spring is linear, the SEA is very efficient in powering the knee joint because it can be designed to take advantage of the linear torque-angle (or spring-like) relationship of the knee in the early stance flexion-extension 


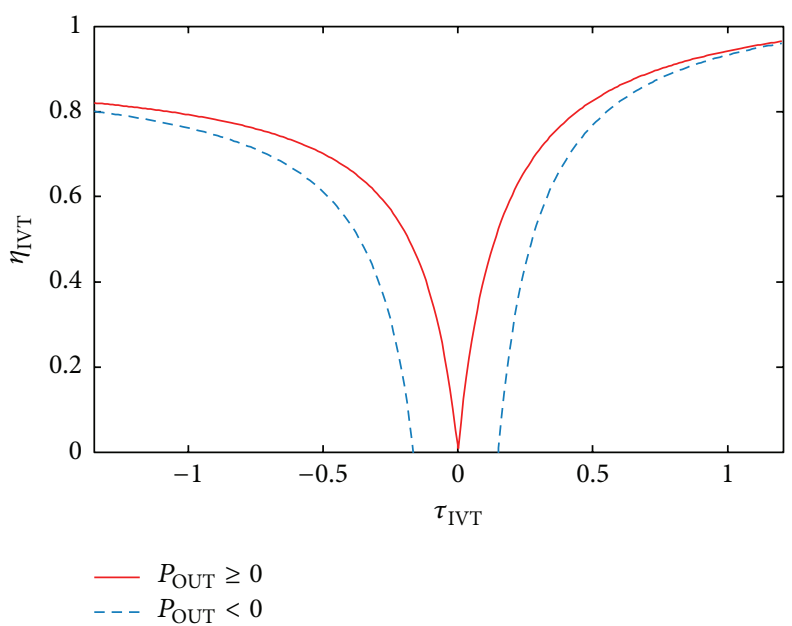

(a)
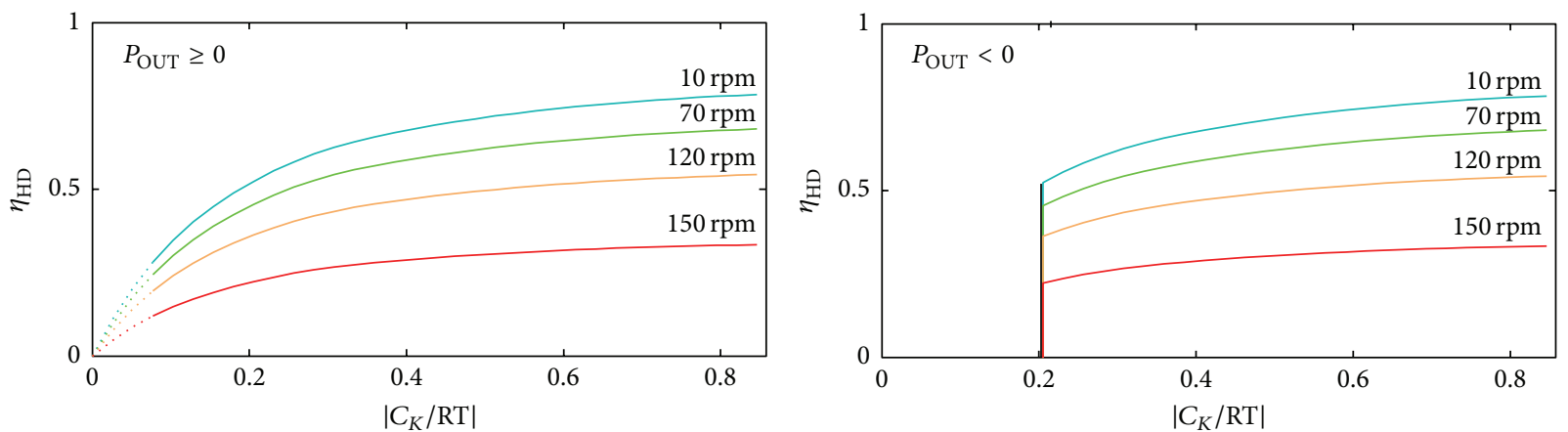

(b)

FIGURE 3: Efficiency maps of the IVT (a) and of the HD gear (b) obtained through detailed efficiency models which distinguish the performance of the transmission devices in forward $\left(P_{\text {OUT }} \geq 0\right)$, revrse $\left(P_{\text {OUT }}<0\right)$, and irreversible motion conditions. In (a) $\eta_{\text {IVT }}$ is given as a function of $\tau_{\text {IVT }}$ whereas $\eta_{\mathrm{HD}}$ is given as a function both of the output motor speed and of the torque at the output shaft normalized to the Rated Torque (RT) of the HD gear selected.

phase of level ground walking. In particular if the stiffness of spring $(K)$ is chosen equal to the slope of the torqueangle curve, the spring alone would render the spring-like relationship, resulting in a great reduction of the motor mechanical energy requirement.

In this work the electric power and energy requirement of SEA to power knee joint in level ground walking at different speeds and in stairs climbing at different inclinations are calculated through (3)-(4) as a function of angle $\left(\vartheta_{M}=\right.$ $\left(C_{K} / K+\vartheta_{K}\right)\left(1 / \tau_{\mathrm{GT}}\right), \tau_{\mathrm{GT}}$ being speed ratio of the gear train) and torque $\left(C_{M}=C_{K} \tau_{\mathrm{GT}} / \eta_{\mathrm{GT}}, \eta_{\mathrm{GT}}\right.$ being efficiency of the gear train) requirements of the SEA motor.

As done for the F-IVT, the SEA was also sized to power the knee joint even under the most powerful condition examined and it was optimized according to the most frequent one. In particular the most powerful condition leads to the choice of both the motor and the HD, whereas the most frequent one leads to the choice of series stiffness which minimizes the energy consumption. In our calculations we used the same walking cycles of [17] so also the optimal value of the stiffness coefficient of the spring is the one determined in [17].
According to [24], the gear train of the SEA comprises a harmonic drive gear (HD). The value of gear ratio of the HD is larger for SEA than for the F-IVT to reduce the maximum torque value requested to the motor and thus to limit the motor size. This choice leads to an average efficiency of the $\mathrm{HD}$ that is smaller than that in the F-IVT. The selected $400 \mathrm{~W}$ brushless Maxon Motor (model: EC 60 p.n. 167131) is able to provide the maximum torque requested to the motor $\left(C_{M}^{\max }=1.494 \mathrm{Nm}\right)$ while working under the motor speed limit $\left(\omega_{M}^{\max }=7322 \mathrm{rpm}\right)$. We noticed that this motor is too heavy for a proficient use in a wearable device, but, on the other hand, it is the only one allowing the SEA to power the knee in all the conditions examined. The model parameters adopted are listed in Table 1.

2.3. Clutchable Series Elastic Actuator (C-SEA). The C-SEA (Figure 5(b)) was developed to overtake one limit of SEAs related to the inefficiency of the motor in the stance phase [7]. In fact, even if SEAs greatly reduce the motor mechanical energy requirement in the stance phase when the spring alone 
TABLE 1: Model parameters of the actuators F-IVT, SEA, and C-SEA. The motor damping parameters and the Coulomb friction torque are not provided by data sheet, but they were estimated following the guidelines given by Maxon.

\begin{tabular}{|c|c|c|c|c|}
\hline & & F-IVT & SEA & C-SEA \\
\hline \multirow{9}{*}{ Brushless motor } & Model & $\begin{array}{c}\text { EC-max } 40 \\
283871\end{array}$ & $\begin{array}{l}\text { EC } 60 \\
167131\end{array}$ & $\begin{array}{c}\text { EC } 45 \\
136209\end{array}$ \\
\hline & $C_{\mathrm{fr}}[\mathrm{mNm}]$ & 5.2 & 0.8 & 0.77 \\
\hline & $J_{M}\left[\mathrm{~g} \mathrm{~cm}^{2}\right]$ & 101 & 831 & 209 \\
\hline & $k_{\mathrm{emf}}[\mathrm{V} / \mathrm{rad} / \mathrm{s}]$ & 0.0628 & 0.147 & 0.0739 \\
\hline & $k_{t}[\mathrm{mNm} / \mathrm{A}]$ & 62.8 & 147 & 73.9 \\
\hline & $R[\Omega]$ & 2.02 & 1.03 & 1.01 \\
\hline & $v[\mathrm{Nm} / \mathrm{rad} / \mathrm{s}]$ & $8.66 \cdot 10^{-6}$ & $1.4 \cdot 10^{-4}$ & $2.7 \cdot 10^{-5}$ \\
\hline & $i_{C}[\mathrm{~A}]$ & - & & 0.25 \\
\hline & $v_{C}[\mathrm{~V}]$ & - & & 24 \\
\hline Flywheel & $J_{v}\left[\mathrm{~g} \mathrm{~cm}^{2}\right]$ & $5 \cdot 10^{3}$ & - & - \\
\hline IVT & $\tau_{\text {IVT }}$ & {$[-1.35,1.21]$} & - & - \\
\hline \multirow{2}{*}{ Gear train } & $\tau_{\mathrm{GT}}$ & $1 / 50$ & $1 / 80$ & $1 / 143$ \\
\hline & $\eta_{\mathrm{GT}}$ & By model & By model & 0.9 \\
\hline Spring & $K[\mathrm{Nm} / \mathrm{rad}]$ & - & 375.14 & 250 \\
\hline
\end{tabular}

powers the knee joint, the electric motor works at very slow speeds and thus very inefficiently. The C-SEA improved the design of SEAs by including a clutch on the motor shaft that is conveniently activated or deactivated according to the cycle phase. In particular during the spring-like phase of the gait cycle the clutch is activated and the spring renders the complete torque-angle relationship while the motor is required to supply only reactionary torque; during the other phases of the cycle, the clutch is deactivated and the C-SEA works as a SEA.

Similar equations as those of SEAs govern the operation of the C-SEA. The complete set of equations given in [7] follows:

$$
\begin{aligned}
& C_{M}= \begin{cases}\frac{C_{K} \tau_{\mathrm{GT}}}{\eta_{\mathrm{GT}}} & \text { clutch off } \\
0 & \text { clutch on, }\end{cases} \\
& \vartheta_{M}= \begin{cases}\left(\frac{C_{K}}{K}+\vartheta_{K}\right) \frac{1}{\tau_{\mathrm{GT}}} & \text { clutch off } \\
0 & \text { clutch on, }\end{cases} \\
& i_{M} \\
& = \begin{cases}\frac{C_{M}}{k_{t}}+\frac{J_{M} \dot{\omega}_{M}}{k_{t}}+\frac{\nu \omega_{M}}{k_{t}}+\frac{\operatorname{sign}\left(\omega_{M}\right) C_{\mathrm{fr}}}{k_{t}} & \text { clutch off } \\
i_{C} & \text { clutch on, }\end{cases} \\
& v_{M}= \begin{cases}R i_{M}+k_{\mathrm{emf}} \omega_{M} & \text { clutch off } \\
v_{C} & \text { clutch on, }\end{cases}
\end{aligned}
$$

where $i_{C}$ is the clutch current and $v_{C}$ is the clutch voltage when the clutch is activated.

In this work the authors estimated the electric energy and power consumption of the C-SEA in level ground walking at different speeds and in stairs climbing at different inclinations. The mathematical model and many of the parameters of [7] were adopted to calculate the performance of C-SEA. However, because of the different reference gait cycle data some characteristics have been changed and a bigger electric motor was selected. A $250 \mathrm{~W}$ brushless Maxon Motor (model: EC 45 p.n. 136209, parameters in Table 1) was chosen because it is able to provide a peak torque compatible with the maximum required $\left(C_{M}^{\max }=0.323 \mathrm{Nm}\right)$ in the locomotion regime explored and the maximum motor speed $\left(\omega_{M}^{\max }=11574 \mathrm{rpm}\right)$ is always below the motor speed limit. The optimal spring stiffness value $(K)$ was also not the same as [7] because of the different gait cycles data considered in this work. In particular, the optimal spring stiffness in [7] is defined as the one which minimizes the kinematic similarity defined as

$$
\Psi=\int\left(\vartheta_{\mathrm{CSEA}}-\vartheta_{K}\right)^{2} d t
$$

where $\vartheta_{\text {CSEA }}$ is the output displacement of the C-SEA and $\vartheta_{K}$ is the output displacement of the knee joint. Such criterion was adopted by Rouse et al. in [7] because they proved that the stiffness of the spring does not affect the electrical power profiles but determines very different knee kinematics. Indeed, when the stiffness affects the knee angle displacement, the clutch absorbs a nearly constant electrical power. Based on this minimization criterion, we calculated an optimal stiffness value of $250 \mathrm{Nm} / \mathrm{rad}$, at which the kinematic agreement was of $8 \cdot 10^{-4} \mathrm{rad}^{2} \mathrm{~s}$ (Figure 6). 


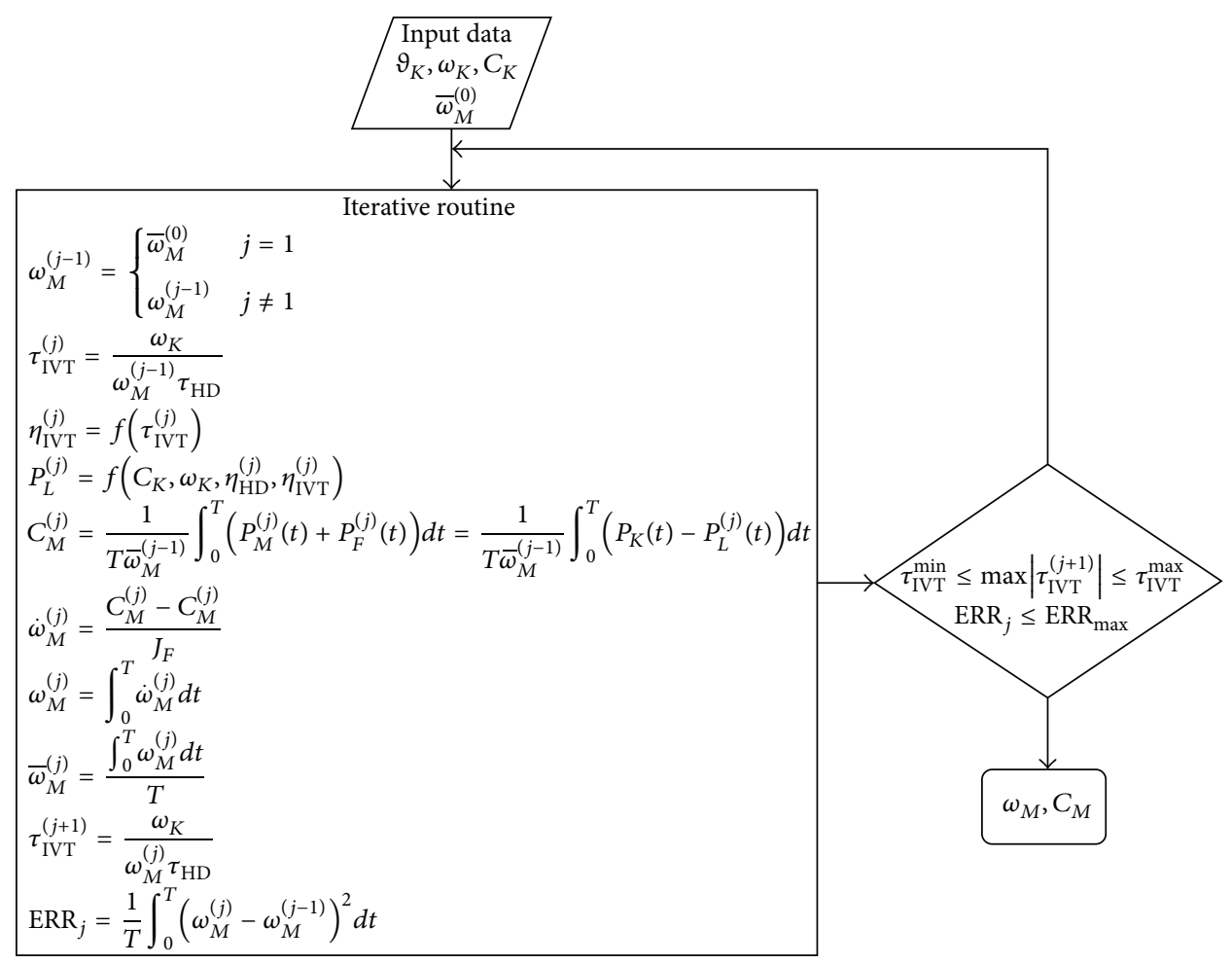

FIGURE 4: Schematic representation of the iterative routine to solve to calculate the mechanical motor requirements $\left(\omega_{M}, C_{M}\right)$.

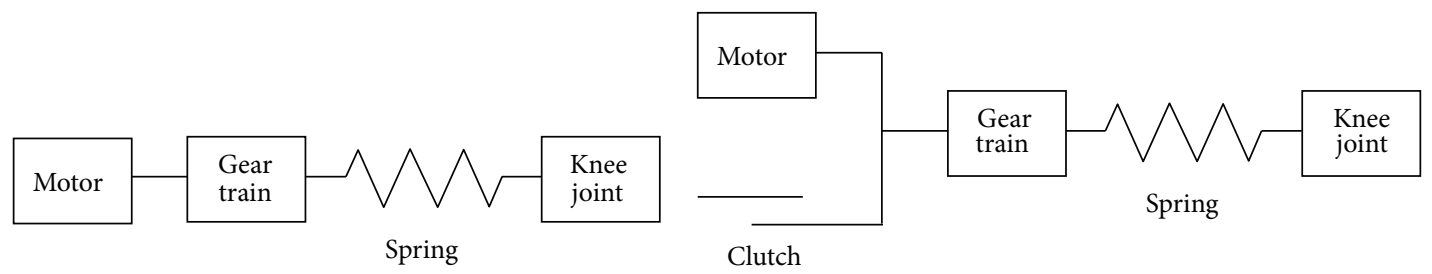

(a) SEA

(b) C-SEA

FIgURE 5: Schematic pictures of the SEA (a) and C-SEA (b).

\section{Results and Discussion}

The electric power and energy consumption of F-IVT, SEA, and C-SEA actuators were estimated through the models described in Section 2, following an inverse dynamic approach. All the models parameters are listed in Table 1. Representative weight-normalized human locomotion data $[17,18]$ were given as input to the models to estimate the performance of all the actuators in powering the knee joint of a $75 \mathrm{~kg}$ person walking at different speeds and climbing stairs of different inclinations.

The electric energy demanded by the motor and the electric power profile result from the simulations done. These quantities are significant performance indexes for the development of lightweight and portable wearable robots because they affect the size and the weight of the actuator, as well as the operating range of the device.
The motor generation of electric energy has been usually neglected in the literature, because the efficiency of regeneration was considered to be too small. Only recently some researchers claimed that at the present state of the art the electric motors are enough efficient to permit a proficient use of the regenerative braking also in biomechatronic devices [7]. Indeed, although mathematical model of the brushless motor (Section 2.1) predicts very large values of the efficiency of the electric motor in the 2nd and 4th quadrant, experimental measurements are only in qualitative agreement with the model, since the measured values of efficiency are in the range $18-30 \%$ [25]. Therefore, two different estimations of the electric energy requirement were carried on in this work for SEA and C-SEA devices: in the first one the possibility of regenerating electric energy is considered; in the second one it is neglected. This distinction does not concern the F-IVT where the electric drive always works as a motor, providing 


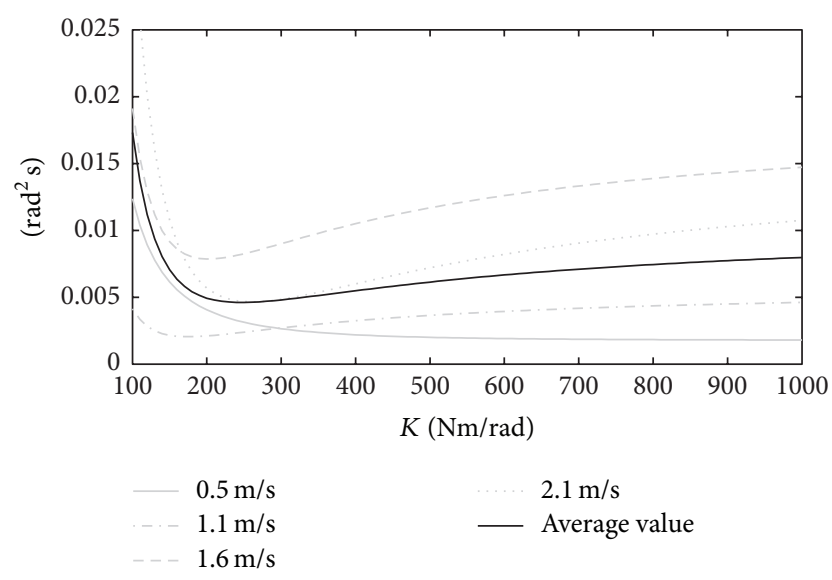

FIGURE 6: Kinematic similarity $(\Psi)$ as a function of series stiffness averaged across walking speeds for subject weight of $75 \mathrm{~kg}$. The minimum value of $\Psi$ is kept approximately at the series stiffness value of $250 \mathrm{Nm} / \mathrm{rad}$.

a positive amount of energy in the whole gait cycle at all the walking speeds and at all the stairs inclinations.

When the electric energy requirement of the F-IVT is compared with the electric energy requirements of SEA and C-SEA (Figure 7) two cases must be distinguished: SEA and C-SEA with regenerative braking and with no regenerative braking. In the former case (Figures 7(a) and 7(b)) the F-IVT is the most power-demanding device at all the conditions of walking and stairs ascending. The C-SEA is the only one actuator with a total negative energy requirement at all the level ground walking speeds, and this is due to the greater efficiency of the transmission devices when compared to efficiencies of the SEA. In fact, even if the motor of SEA generates electric energy when the motor power is negative, as a consequence of the low value of $\eta_{\mathrm{HD}}$, which is often such to prevent the back drivability of the system, the total energy required by the motor is always positive for SEA.

In the no-regenerative braking case, the SEA has the greater electric energy consumption almost at all the walking speeds, whereas the C-SEA is still the most convenient one because of the low motor power absorbed when the clutch is activated (Figure $7(\mathrm{c})$ ).

In stairs climbing the F-IVT always needs more electric energy than the SEA and the C-SEA if the regenerative braking of the motor is either considered or not (Figures 7 (b) and 7(d)), whereas the C-SEA proves to be the most advantageous solution. It can be noticed that there is no evident difference of the values of energy required by SEA and $\mathrm{C}$-SEA in stairs climbing between the regenerative and the nonregenerative cases. In fact, while the total energy required by the knee in level ground walking is negative, it is positive in stairs climbing and thus the possibility of recovering energy is reduced.

The peak of electric power is another important factor to estimate the performance of an actuator for lower limb joints, because it affects the size and the weight of the motor (Figure 8). The F-IVT permits greatly reducing the electric peak power at all the locomotion conditions, if the regenerative braking of the motor is either considered or not. In fact one of the achievements of the F-IVT is to stabilize the working point of the motor, which provides a nearly constant amount of power whose value is close to the mean value of the power request and by far less than the peak (Figure 9). On the contrary, the motor of both the SEA and C-SEA has to follow the torque request of the knee joint, supplying variable power. This is clearly shown in Figure 9 where the instant value of motor power is shown as a function of time for all the devices under analysis and in two walking regimes, for instance, walking at $2.1 \mathrm{~m} / \mathrm{s}$ (Figure 9(a)) and stairs climbing at inclination of $30^{\circ}$ (Figure $8(\mathrm{~b})$ ). Similar results have been obtained in all other walking regimes.

From these results the F-IVT seems to be the most effective solution for reducing the peak power of the motor, whereas the C-SEA is more energy efficient in all the conditions examined, if the regenerative braking is either permitted or not. On one hand, the F-IVT would permit a consistent electric motor downsizing; on the other hand, CSEA would permit reducing the electric energy demanded by the motor for a given working range of the device. However, the gap between the C-SEA and the F-IVT in terms of energy consumption is considerably larger in the case of regenerative C-SEA, where our calculations may be affected by an overestimation of the electric energy recovered by regenerative braking with respect to the value achievable in practice [23].

Furthermore, the power losses in all electric modules may also alter the comparison of energy consumption of F-IVT and C-SEA (or SEA), and they have not been considered in this study. Indeed, all electronic modules and also the batteries would work differently in the F-IVT and in C-SEA (or SEA): in one case the electric power is almost constant with constant angular velocity of the motor; in the other cases both the power and the velocity of the motor vary periodically. Therefore, the total energy consumption of the actuators, that is, the energy requirement of the battery, may be quite different from the electric energy demanded by the motor.

\section{Conclusions}

In this work the authors compared the motor performance of three different actuators, F-IVT, SEA, and C-SEA, powering an artificial knee joint under different locomotion regimes very recurring in daily life: level ground walking at different speeds and stairs climbing at different inclinations. Detailed efficiency models of all the devices were developed to obtain realistic estimations of the motor consumptions following an inverse dynamic approach.

Two main performance indexes have been taken into consideration for comparison: the energy consumption (per gait) and the peak of electric power, both calculated at motor electrodes.

As a result of the simulations, it was found that the motor of the C-SEA has an electric energy consumption which is always lower compared to the F-IVT and the SEA at all the locomotion regimes considered. Thus the C-SEA would permit a larger operating range of the device. However, it is important to specify that in this work the energy requirement 


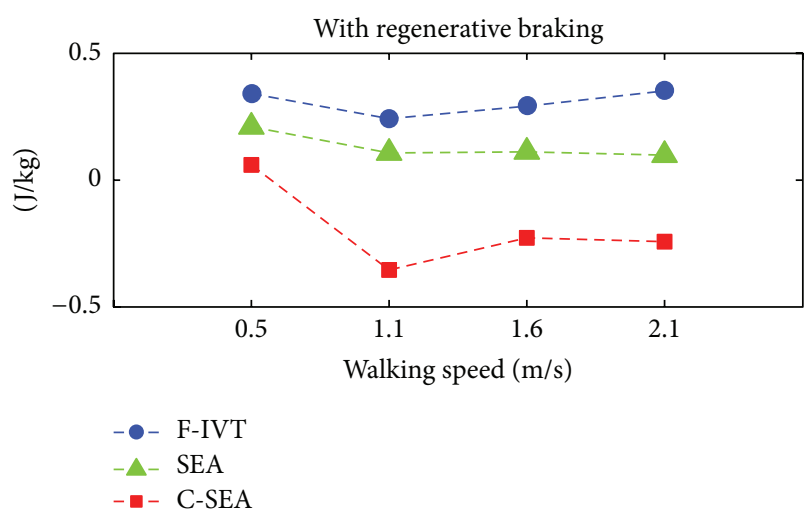

(a)

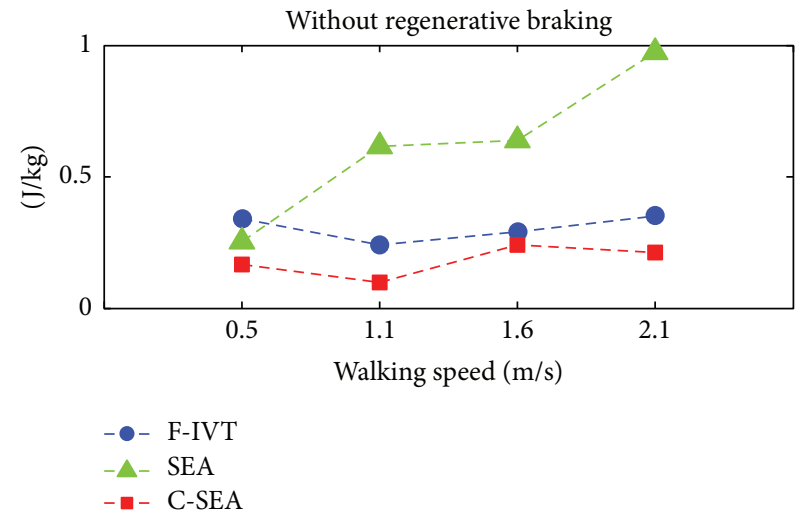

(c)

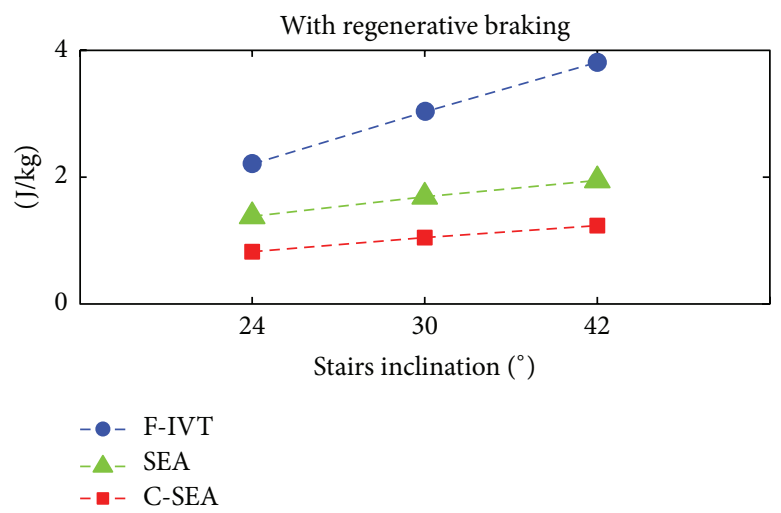

(b)

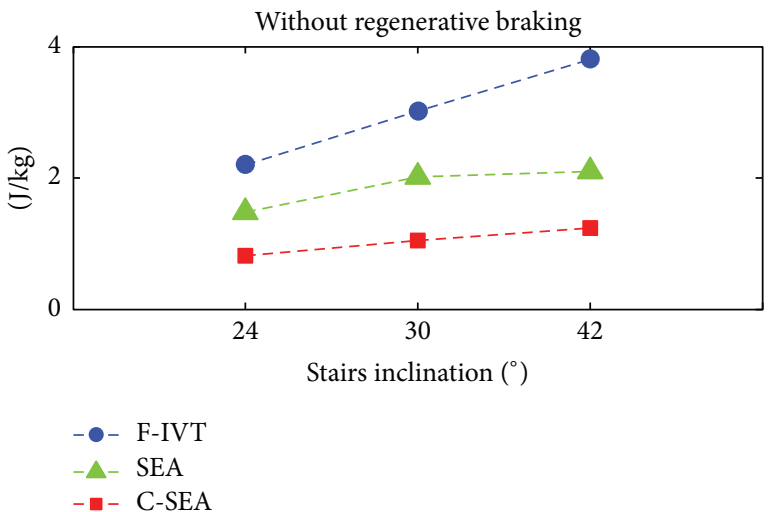

(d)

FIGURE 7: Motor electric energy requirement of the actuators compared (F-IVT, SEA, and C-SEA) in level ground walking at different speeds $(a, c)$ and in stairs climbing at different inclinations (b, d), under the assumptions of regenerative braking admitted (a,b) and not admitted $(c, d)$.

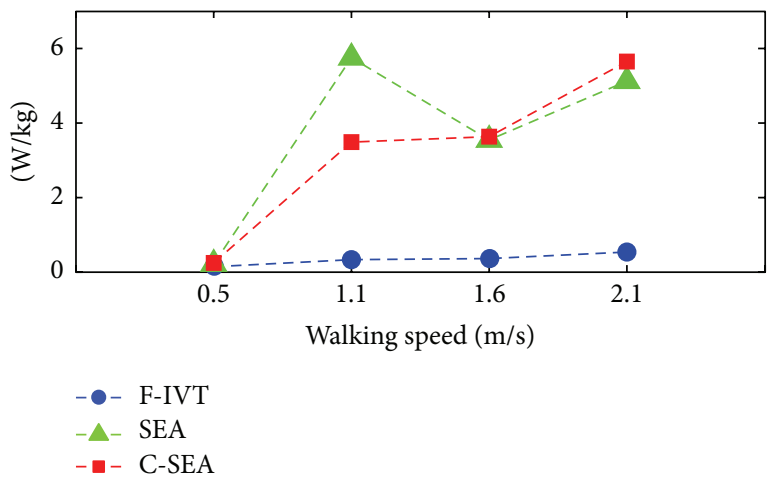

(a)

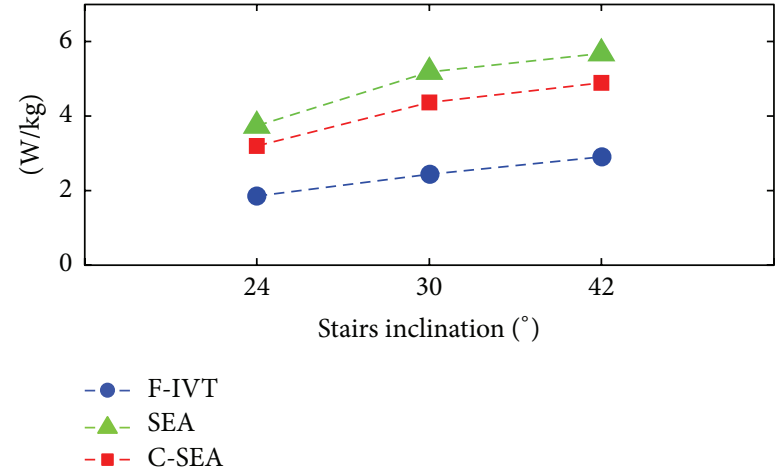

(b)

FIGURE 8: Electric peak power of the motors of the actuators compared (F-IVT, SEA, and C-SEA) in level ground walking at different speeds (a) and in stairs climbing at different inclinations (b).

of the motor has been calculated at the motor electrodes, not at batteries. There are at least two reasons why the effective energy requirement of the actuators, that is, the energy demanded by the battery, may be different with respect to the motor energy requirement, in particular in SEA and C-SEA. First, the working point of the motor is quickly and repeatedly variable, and this may affect the actual efficiency of the electronic devices between the batteries and the motor and the batteries themselves. Second, the electrical power generated by the motor could be smaller than the estimated, as the very low experimental values of the regeneration efficiency achieved in practice [23] would 


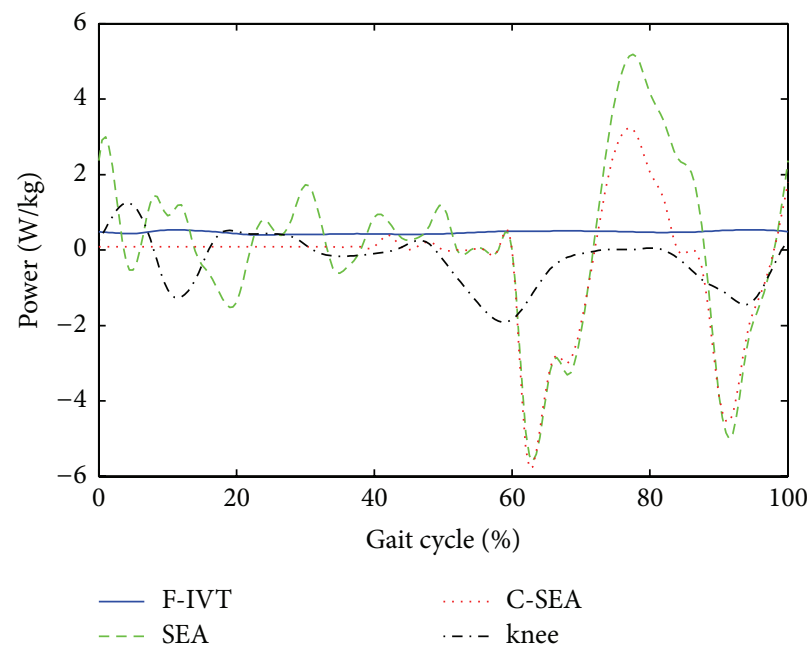

(a)

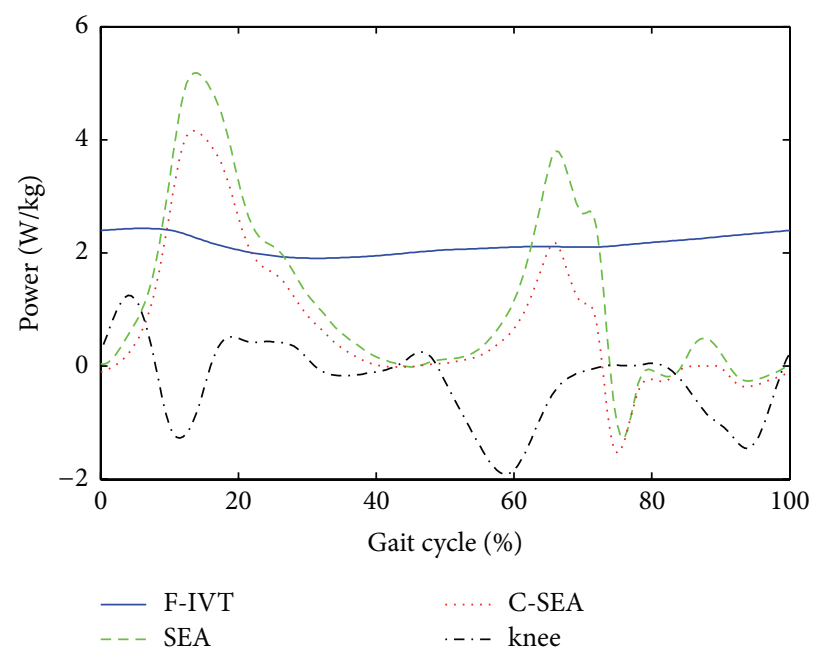

(b)

FIGURE 9: Instant power requirements of the knee joint and of the electric motor of the actuators compared (F-IVT, SEA, and C-SEA) in level ground walking at $2.1 \mathrm{~m} / \mathrm{s}$ (a) and in stairs climbing at inclination of $30^{\circ}$ (b).

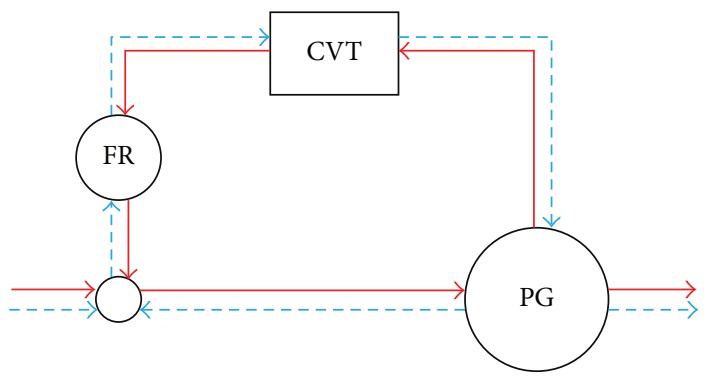

- Type I

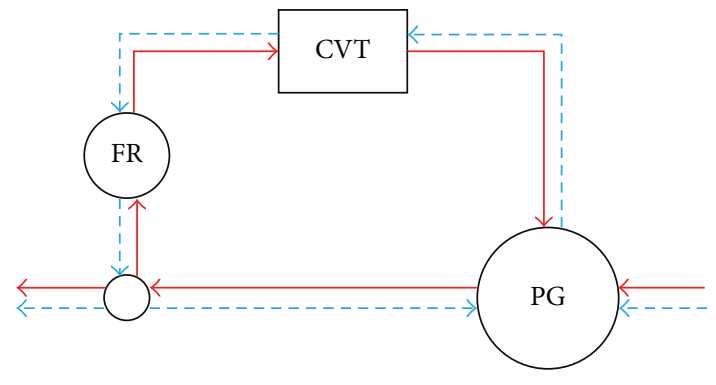

- Type I

(a)

(b)

FIGURE 10: Schematic picture of the shunted CVT architecture of IVT, where power circulation of type I or that of type II can take place. Figures above depict the two types of power circulation in direct (a) and in reverse (b) operating modes.

demonstrate. Unfortunately, these issues are still debated, and no precise data are available in the literature.

Furthermore, in this study it was also proved that the FIVT permit achieving a drastic reduction of the peak power at all the conditions examined. In fact, the flywheel and the IVT stabilize the working point of the motor, which always provides a nearly constant amount of power that is very lower than the peak power request. This achievement is very important in view of the possibility of downsizing the electric motor, which would also mean reducing the size and the weight of the actuator, with advantages in terms of portability. Of course, even if the motor mass is the most critical one, in order to make the F-IVT more lightweight than the other devices developed for the same purpose, the mass loss due mainly to the reduced motor size should overcome the additional masses of the micro-IVT and the flywheel.
Moreover, the motor of the F-IVT always works very efficiently, even when it does not work under the condition for which it is optimized, and this may be true for the power electronics and the batteries.

The comparison of the three actuators done in this paper aims at evaluating which are the potential benefits or the drawbacks of the F-IVT actuator, in order to evaluate if it could or could not be a competitive actuator for artificial knee joints of wearable robots. Furthermore, the results of the comparison are usefully supporting the development of a prototype of the F-IVT in which the authors are involved. For example, a careful analysis of the results obtained in this work reveals that at the present state of the art the weak point of the F-IVT is the overall efficiency of the driveline, the bottleneck of which is the harmonic drive. These results stimulate to find alternative possibilities in order to improve the efficiency of F-IVT to reduce the motor energy requirement, keeping its indisputable advantages safe. 


\section{Appendix}

\section{A. Shunted CVT Architecture}

Among the different typologies of IVT proposed in literature [26-31], the most popular one is the shunted CVT architecture (Figure 10) which includes the following component devices: a microtoroidal CVT (Continuously Variable Transmission), PG (micro planetary gear train), and FR (fixed speed ratio drive). An IVT with shunted CVT architecture was considered in the F-IVT actuator, because mathematical models of its efficiency are well established in the literature [20].

An IVT is needed in the actuation system of lower limb joints because it is able to continuously change its speed ratio between positive and negative values, permitting the motor speed to be kept constant or nearly constant, while the joint always works at the desired speed value. The IVT speed ratio is given as a function of the transmission ratios of all the components of the IVT:

$$
\tau_{\mathrm{IVT}}=\frac{1+\tau_{\mathrm{CVT}} \tau_{\mathrm{FR}} X}{1+X},
$$

where $\tau_{\mathrm{FR}}$ is the speed ratio of the FR and $X$ is the basic transmission ratio of the PG.

Very often the lower and upper bounds of $\tau_{\mathrm{CVT}}$ are fixed, being an intrinsic geometric feature of the device. The IVT under consideration includes a microtoroidal CVT whose ratio range varies between 0.4 and 2.5. The speed ratio range of the IVT has also to be fixed to match the requirements of the load (Table 1). Once the lower and upper bounds of $\tau_{\mathrm{IVT}}$ and $\tau_{\mathrm{CVT}}$ are chosen, both $\tau_{\mathrm{FR}}$ and $X$ can be calculated through (A.1).

The IVT enlarges the transmission ratio range of the CVT $\left(\tau_{\mathrm{CVT}}\right)$, but this increase involves a power circulation in the IVT device, which can be reverse or forward according to the relationship between $\tau_{\mathrm{IVT}}$ and $\tau_{\mathrm{CVT}}$. The reverse power circulation, which is named power flow of type $\mathrm{I}$, is characterized by a monotonic decrease of $\tau_{\mathrm{IVT}}$ when $\tau_{\mathrm{CVT}}$ increases; conversely the forward power circulation, named power flow of type II, is characterized by a monotonic increase of $\tau_{\text {IVT }}$ when $\tau_{\mathrm{CVT}}$ increases. The IVT with type I power flow for positive value of the $\tau_{\text {IVT }}$ and with type II power flow for the negative ones was chosen as the most adequate for our purposes.

The efficiency of the IVT ( $\left.\eta_{\text {IVT }}\right)$ was modeled as in [20], considering the different operating modes of the transmissions, that is, the direct and the reverse mode, and the conditions under which the IVT is not back drivable. $\eta_{\text {IVT }}$ is affected by the type of power circulation both in direct and in reverse operating mode. The efficiency of the IVT is estimated through the following equations:
Direct mode is as follows:

$$
\begin{aligned}
& \eta_{\mathrm{IVT}} \\
& = \begin{cases}\frac{1+\tau_{\mathrm{CVT}} \tau_{\mathrm{FR}} X}{1+\tau_{\mathrm{CVT}} \tau_{\mathrm{FR}} X \eta_{\mathrm{CVT}} \eta_{\mathrm{FR}}} & \text { Type I }\left(\tau_{\mathrm{IVT}} \geq 0\right) \\
\frac{\eta_{\mathrm{CVT}} \eta_{\mathrm{FR}}\left(1+\tau_{\mathrm{CVT}} \tau_{\mathrm{FR}} X\right)}{\eta_{\mathrm{CVT}} \eta_{\mathrm{FR}}+\tau_{\mathrm{CVT}} \tau_{\mathrm{FR}} X} & \text { Type II }\left(\tau_{\mathrm{IVT}}<0\right) .\end{cases}
\end{aligned}
$$

Reverse mode is as follows:

$$
\begin{aligned}
& \eta_{\mathrm{IVT}} \\
& = \begin{cases}\frac{\eta_{\mathrm{CVT}} \eta_{\mathrm{FR}}+\tau_{\mathrm{CVT}} \tau_{\mathrm{FR}} X}{\eta_{\mathrm{CVT}} \eta_{\mathrm{FR}}\left(1+\tau_{\mathrm{CVT}} \tau_{\mathrm{FR}} X\right)} & \text { Type I }\left(\tau_{\mathrm{IVT}} \geq 0\right) \\
\frac{1+\eta_{\mathrm{CVT}} \eta_{\mathrm{FR}} \tau_{\mathrm{FR}} X \tau_{\mathrm{CVT}}}{1+\tau_{\mathrm{FR}} \tau_{\mathrm{CVT}} X} & \text { Type II }\left(\tau_{\mathrm{IVT}}<0\right) .\end{cases}
\end{aligned}
$$

In our calculations we assumed that $\eta_{\mathrm{CVT}}=0.93$ and $\eta_{\mathrm{FR}}=$ 0.98 . $\eta_{\mathrm{CVT}}$ was assumed to be constant for the reasons given in [14].

The efficiency curves of the IVT shown in Figure 3(a) are obtained through (A.2)-(A.3). It can be noticed that $\eta_{\text {IVT }}$ is larger in forward than in reverse operating mode in the whole range of $\tau_{\text {IVT }}$. Moreover $\eta_{\text {IVT }}$ gets its maximum value at the boundary values of the IVT ratio range and decreases when the absolute value of $\tau_{\text {IVT }}$ becomes small, close to the neutral gear condition $\left(\tau_{\text {IVT }}=0\right)$. This is why the ratio range was optimized according to the speed range of the knee joint to improve the global usage efficiency of the IVT. It can also be noticed that the back drive ability of the transmission is not permitted for very low values of $\left|\tau_{\text {IVT }}\right|\left(\tau_{\text {IVT }} \in[-0.16,0.14]\right)$.

\section{B. Sizing Procedure of the F-IVT}

In this work the F-IVT was sized according to the task requirements of the actuator: walking at different speeds and stairs climbing at different inclinations. To design an efficient F-IVT, both the most powerful and the most frequent working conditions must be considered. In particular, the former defines the size of the HD gear and of the motor, whereas the latter (level ground walking at $1.1 \mathrm{~m} / \mathrm{s}$ ) is necessary to optimally design the F-IVT in such a way that it can work very efficiently as often as possible. The optimal design of the F-IVT leads to the choice of the HD gear, the ratio range of the IVT, and the related value of the motor working speed. Indeed, as it will be clarified later, the optimization of the IVT consists in the calculation of its optimal speed ratio range, which also has a direct influence on the actual working point of the motor.

The sizing procedure of the F-IVT started with the selection of the HD gear following the guidelines suggested by one manufacturer (Harmonic Drive AG), which mainly refers to the values of output torque and speed. Because the speed limit is by far respected (the knee speed is always lower than the maximum speed allowed), the HD gear was chosen constraining the greatest value of the output torque in all the gait cycles examined (i.e., stairs climbing at the maximum inclination), to be less than or equal to the Repeatable Peak 
Torque (RPT) value of the HD gear $\left(\max \left|C_{K}\right| \leq \mathrm{RPT}\right)$. The selection of the HD gear focuses on the smallest size and the greatest efficiency, mainly depending on the speed of the input shaft and on the torque of the output shaft, by means of a coefficient that is a function of the Rated Torque (RT) value of the device (Figure 3(b)). Among all the admissible options of $\mathrm{HD}$ gears (i.e., the ones that satisfy $\max \left|C_{K}\right| \leq \mathrm{RPT}$ ), the smallest one with the smallest $\mathrm{RT}$ value is chosen.

The second step is to choose the best motor for the required task. In the F-IVT the motor provides nearly the mean value of the power required by the load in a cycle, thanks to the effect of the flywheel. The flywheel inertia is determined in advance, as a compromise between its advantageous storage and regularizing function on one hand and its weight and size [14] on the other hand.

The motor nominal power must be approximately equal to the maximum value of the mean power demanded in all the working conditions explored. Unfortunately, the average power demanded per cycle is affected also by the efficiency of the IVT which has not yet been determined at this step of the design procedure. In order to overcome this problem, once the HD gear and the flywheel have been selected, the power requirement at the output shaft of the IVT is known and, thus, by assuming guess constant values of $\eta_{\text {IVT }}$ both in direct $\left(\eta_{\mathrm{IVT}}=0.6\right)$ and in reverse motion $\left(\eta_{\mathrm{IVT}}=\right.$ 0.5 ), the mean value of the power requested to the motor in all the conditions was estimated. As it can be expected, the most powerful condition among those examined defines the size of the motor. In our case, we found the following motor for our application: $120 \mathrm{~W}$ brushless Maxon Motor, model EC-max 40 p.n. 283871. After having chosen a suitable motor for the application, the efficiency map of the motor is known, which is a necessary ingredient for the last step of the actuator design. The selected motor is able to provide greater torque and speed than the ones strictly necessary. Peak values of torque and speed are $C_{M}^{\max }=0,165 \mathrm{Nm}$ and $\omega_{M}^{\max }=10677 \mathrm{rpm}$, respectively.

Design of the IVT proceeds in order to minimize the consumption of electric energy at the most frequent gait cycle (level ground walking at $1.1 \mathrm{~m} / \mathrm{s}$ ). The calculation of the optimal values of $\tau_{\mathrm{IVT}}^{\mathrm{min}}$ and $\tau_{\mathrm{IVT}}^{\mathrm{max}}$ needs an iterative procedure. Since the output speed of IVT is known for a given walking regime and for a given HD gear ratio, by assuming that the motor speed is constant and equal to an initial guess value $\omega_{M}^{(0)}$, which belongs to the motor speed working range, then $\tau_{\text {IVT }}$ can be calculated as a function of time as $\omega_{K} /\left(\omega_{M}^{(0)} \tau_{\mathrm{HD}}\right)$ and also $\tau_{\mathrm{IVT}}^{\min }$ and $\tau_{\mathrm{IVT}}^{\max }$ can be determined. It follows (see Appendix A) that also the efficiency of the IVT is known as a function of $\tau_{\text {IVT }}$. Full simulation of the actuator with these parameters leads to the calculation of the actual trend of the motor speed $\omega_{M}^{(1)}$, which does not correspond to the initial guess value. An iteration error (at iteration 0) is defined as $\operatorname{ERR}_{0}=(1 / T) \int_{0}^{T}\left(\omega_{M}^{(1)}-\omega_{M}^{(0)}\right)^{2} d t$. Then the procedure is repeated, starting from $\omega_{M}^{(1)}$ obtained at iteration 0 and so on. The error at iteration $j$ is defined as $\operatorname{ERR}_{j}=(1 / T) \int_{0}^{T}\left(\omega_{M}^{(j+1)}-\omega_{M}^{(j)}\right)^{2} d t$ and the iterations are stopped when $\mathrm{ERR}_{j}<\mathrm{ERR}_{\text {max }}$, where $\mathrm{ERR}_{\text {max }}$ is a proper error tolerance. After iterations are stopped, then the values of $\tau_{\mathrm{IVT}}^{\min }$ and $\tau_{\mathrm{IVT}}^{\max }$ are the ones calculated at the last iteration, and the consumption per cycle can also be calculated.

If the iterative routine is started from a different value of $\omega_{M}^{(0)}$, then different values of $\tau_{\mathrm{IVT}}^{\min }$ and $\tau_{\mathrm{IVT}}^{\max }$ and of the energy consumption are obtained. It follows that the optimal values of $\tau_{\mathrm{IVT}}^{\min }$ and $\tau_{\mathrm{IVT}}^{\max }$ are those which correspond to the minimum value of energy consumption achievable with all possible values of $\omega_{M}^{(0)}$ belonging to the working speed range of the electric motor.

\section{Competing Interests}

The authors declare that they have no competing interests.

\section{References}

[1] J. L. Pons, "Front matter," in Wearable Robots: Biomechatronic Exoskeletons, pp. 1-10, John Wiley \& Sons, Chichester, UK, 2008.

[2] R. Bogue, "Robotic exoskeletons: a review of recent progress," Industrial Robot: An International Journal, vol. 42, no. 1, pp. 510, 2015.

[3] L. Mooney and H. Herr, "Continuously-variable series-elastic actuator," in Proceedings of the IEEE 13th International Conference on Rehabilitation Robotics (ICORR '13), pp. 1-6, IEEE, Seattle, Wash, USA, June 2013.

[4] J. M. Donelan, Q. Li, V. Naing, J. A. Hoffer, D. J. Weber, and A. D. Kuo, "Biomechanical energy harvesting: generating electricity during walking with minimal user effort," Science, vol. 319, no. 5864, pp. 807-810, 2008.

[5] D. F. B. Haeufle, M. D. Taylor, S. Schmitt, and H. Geyer, "A clutched parallel elastic actuator concept: towards energy efficient powered legs in prosthetics and robotics," in Proceedings of the 4th IEEE RAS \& EMBS International Conference on Biomedical Robotics and Biomechatronics (BioRob '12), pp. 16141619, IEEE, Rome, Italy, June 2012.

[6] V. Luciano, E. Sardini, M. Serpelloni, and G. Baronio, "Analysis of an electromechanical generator implanted in a human total knee prosthesis," in Proceedings of the IEEE Sensors Applications Symposium (SAS 2012), pp. 166-170, Brescia, Italy, February 2012.

[7] E. J. Rouse, L. M. Mooney, and H. M. Herr, "Clutchable serieselastic actuator: implications for prosthetic knee design," The International Journal of Robotics Research, vol. 33, no. 13, pp. 1611-1625, 2014.

[8] P. Cherelle, G. Mathijssen, Q. Wang, B. Vanderborght, and D. Lefeber, "Advances in propulsive bionic feet and their actuation principles," Advances in Mechanical Engineering, vol. 2014, Article ID 984046, 21 pages, 2014.

[9] D. Paluska and H. Herr, "The effect of series elasticity on actuator power and work output: implications for robotic and prosthetic joint design," Robotics and Autonomous Systems, vol. 54, no. 8, pp. 667-673, 2006.

[10] G. A. Pratt and M. M. Williamson, "Series elastic actuators," in Proceedings of the IEEE/RSJ International Conference on Intelligent Robots and Systems, Human Robot Interaction and Cooperative Robots, vol. 1, pp. 399-406, Pittsburgh, Pa, USA, August 1995. 
[11] A. K. Raj, P. D. Neuhaus, A. M. Moucheboeuf, J. H. Noorden, and D. V. Lecoutre, "Mina: a sensorimotor robotic orthosis for mobility assistance," Journal of Robotics, vol. 2011, Article ID 284352, 8 pages, 2011.

[12] L. Flynn, J. Geeroms, R. Jimenez-Fabian, B. Vanderborght, N. Vitiello, and D. Lefeber, "Ankle-knee prosthesis with active ankle and energy transfer: development of the CYBERLEGs Alpha-Prosthesis," Robotics and Autonomous Systems, vol. 73, pp. 4-15, 2015.

[13] F. Bottiglione and G. Mantriota, "Effect of the ratio spread of CVU in automotive kinetic energy recovery systems," Journal of Mechanical Design-Transactions of the ASME, vol. 135, no. 6, Article ID 061001, 2013.

[14] R. Alò, F. Bottiglione, and G. Mantriota, "An innovative design of artificial knee joint actuator with energy recovery capabilities," Journal of Mechanisms and Robotics, vol. 8, no. 1, Article ID 011009, 8 pages, 2015.

[15] J. E. Pratt, B. T. Krupp, C. J. Morse, and S. H. Collins, "The RoboKnee: an exoskeleton for enhancing strength and endurance during walking," in Proceedings of the IEEE International Conference on Robotics and Automation (ICRA '04), vol. 3, pp. 2430-2435, IEEE, May 2004.

[16] A. M. Dollar and H. Herr, "Design of a quasi-passive knee exoskeleton to assist running," in Proceedings of the IEEE/RSJ International Conference on Intelligent Robots and Systems (IROS '08), pp. 747-754, Nice, France, September 2008.

[17] M. Grimmer, M. Eslamy, and A. Seyfarth, "Energetic and peak power advantages of series elastic actuators in an actuated prosthetic leg for walking and running," Actuators, vol. 3, no. 1, pp. 1-19, 2014.

[18] R. Riener, M. Rabuffetti, and C. Frigo, "Stair ascent and descent at different inclinations," Gait \&d Posture, vol. 15, no. 1, pp. 3244, 2002.

[19] A. Zoss and H. Kazerooni, "Design of an electrically actuated lower extremity exoskeleton," Advanced Robotics, vol. 20, no. 9, pp. 967-988, 2006.

[20] F. Bottiglione and G. Mantriota, "Reversibility of power-split transmissions," Journal of Mechanical Design, vol. 133, no. 8, Article ID 084503, 2011.

[21] F. Bottiglione, S. De Pinto, and G. Mantriota, "Infinitely variable transmissions in neutral gear: torque ratio and power recirculation," Mechanism and Machine Theory, vol. 74, pp. 285298, 2014

[22] F. Bottiglione, S. De Pinto, G. Mantriota, and A. Sorniotti, "Energy consumption of a battery electric vehicle with infinitely variable transmission," Energies, vol. 7, no. 12, pp. 8317-8337, 2014.

[23] M. R. Tucker and K. B. Fite, "Mechanical damping with electrical regeneration for a powered transfemoral prosthesis," in Proceedings of the IEEE/ASME International Conference on Advanced Intelligent Mechatronics (AIM '10), pp. 13-18, IEEE, Montreal, Canada, July 2010.

[24] F. Sergi, D. Accoto, G. Carpino, N. L. Tagliamonte, and E. Guglielmelli, "Design and characterization of a compact rotary series elastic actuator for knee assistance during overground walking," in Proceedings of the 4th IEEE RAS \& EMBS International Conference on Biomedical Robotics and Biomechatronics (BioRob '12), pp. 1931-1936, IEEE, Rome, Italy, June 2012.

[25] R. Rarick, H. Richter, A. Van Den Bogert, D. Simon, H. Warner, and T. Barto, "Optimal design of a transfemoral prosthesis with energy storage and regeneration," in Proceedings of the American
Control Conference (ACC '14), pp. 4108-4113, Portland, Ore, USA, June 2014.

[26] M. Bazyn, J. Carter, C. B. Lohr, C. Malone, L. T. McDaniel, and P. D. Poxton, "Continuously and/or infinitely variable transmissions and methods therefor," U.S. Patent 8,313,405, U.S. Patent and Trademark Office, Washington, DC, USA, 2002.

[27] L. G. Brown, G. A. Brown, and B. A. Brown, "Locked contact infinitely variable transmission," Patent US8419589 B1, 2013.

[28] C. J. Greenwood, A. D. De Freitas, and A. R. Oliver, "Drive mechanism for Infinitely Variable Transmission," Patent n. US7955210 B2, 2011.

[29] K. Kazerounian and Z. Furu-Szekely, "Parallel disk continuously variable transmission (PDCVT)," Mechanism and Machine Theory, vol. 41, no. 5, pp. 537-566, 2006.

[30] C. B. Lohr, J. W. Sherrill, B. P. Pohl, R. Dawson, and C. Pew, "Infinitely variable transmissions, continuously variable transmissions, methods, assemblies, subassemblies, and components therefor," Patent US8721485 B2, 2014.

[31] M. Douglas, "Infinitely Variable Transmission," Patent n. US7704184 B2, 2010. 


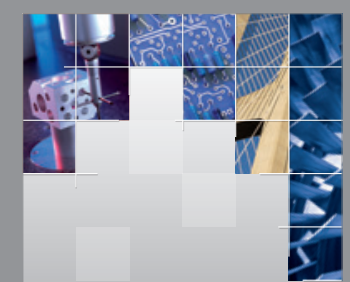

\section{Enfincering}
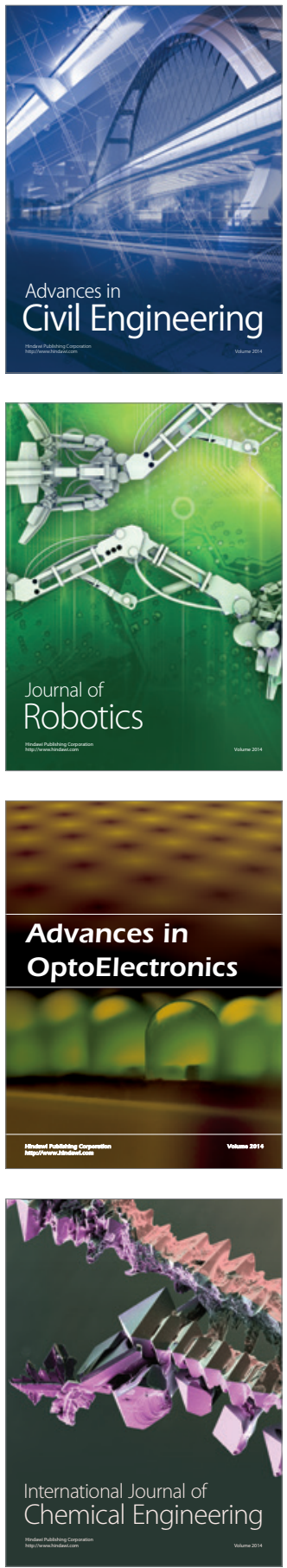

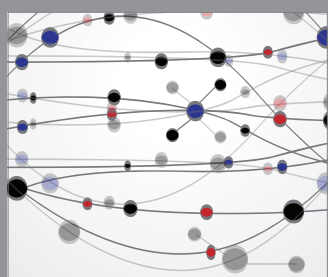

The Scientific World Journal

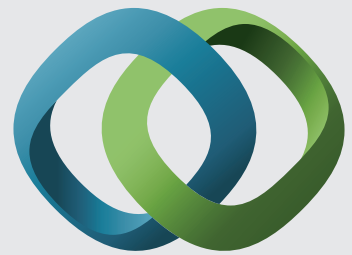

\section{Hindawi}

Submit your manuscripts at

http://www.hindawi.com
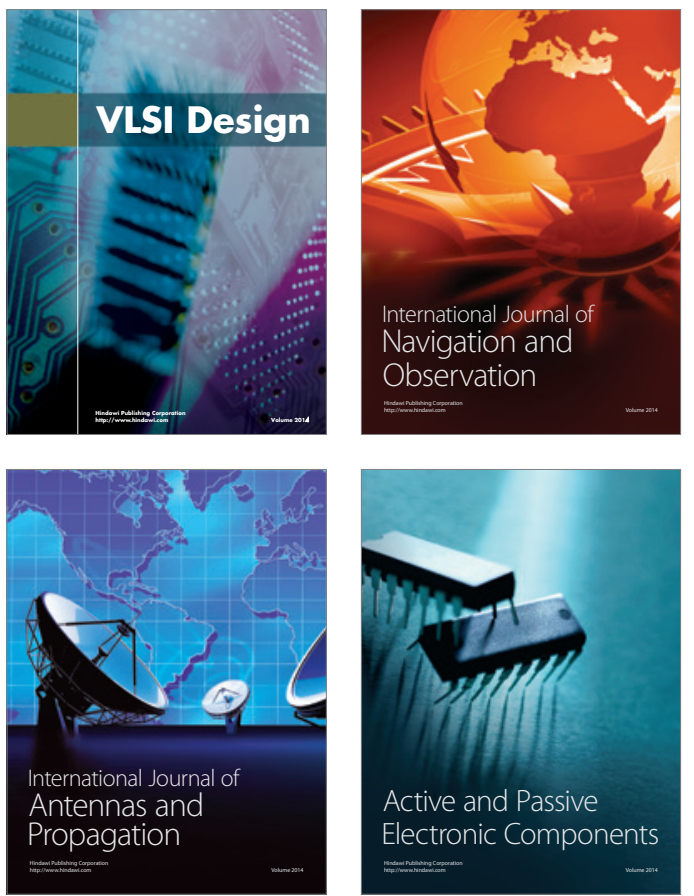
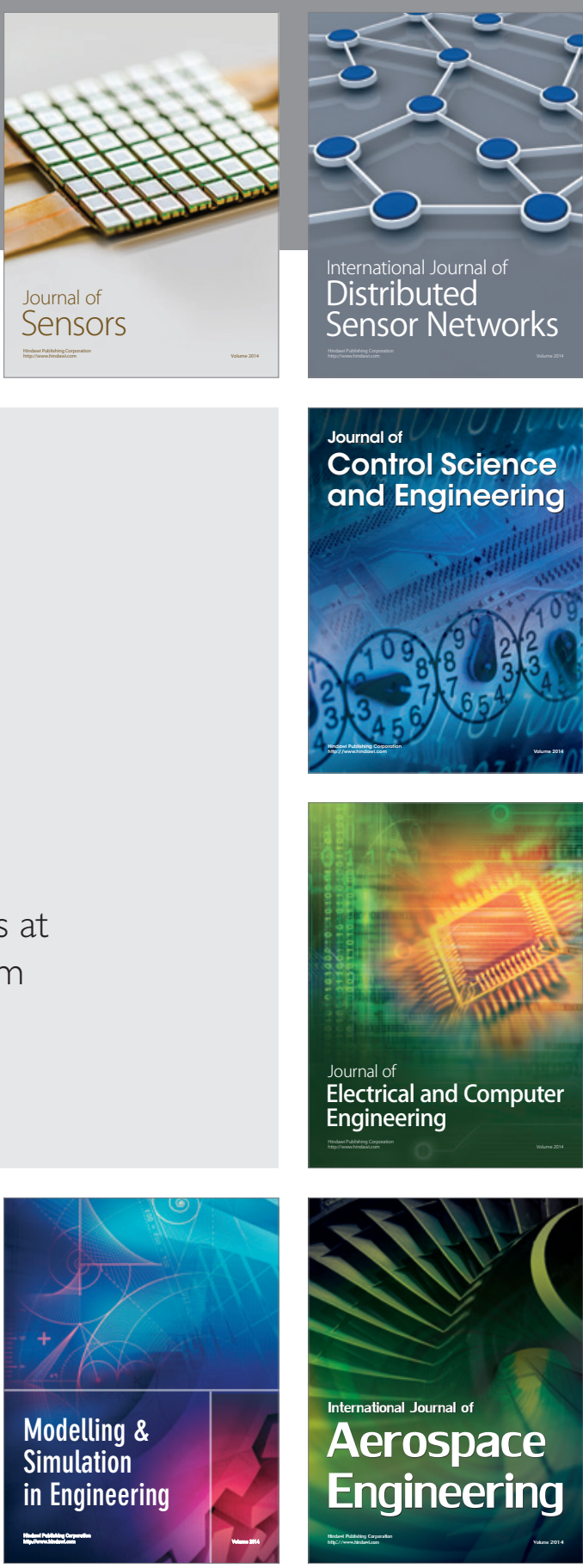

International Journal of

Distributed

Sensor Networks

Journal of

Control Science

and Engineering
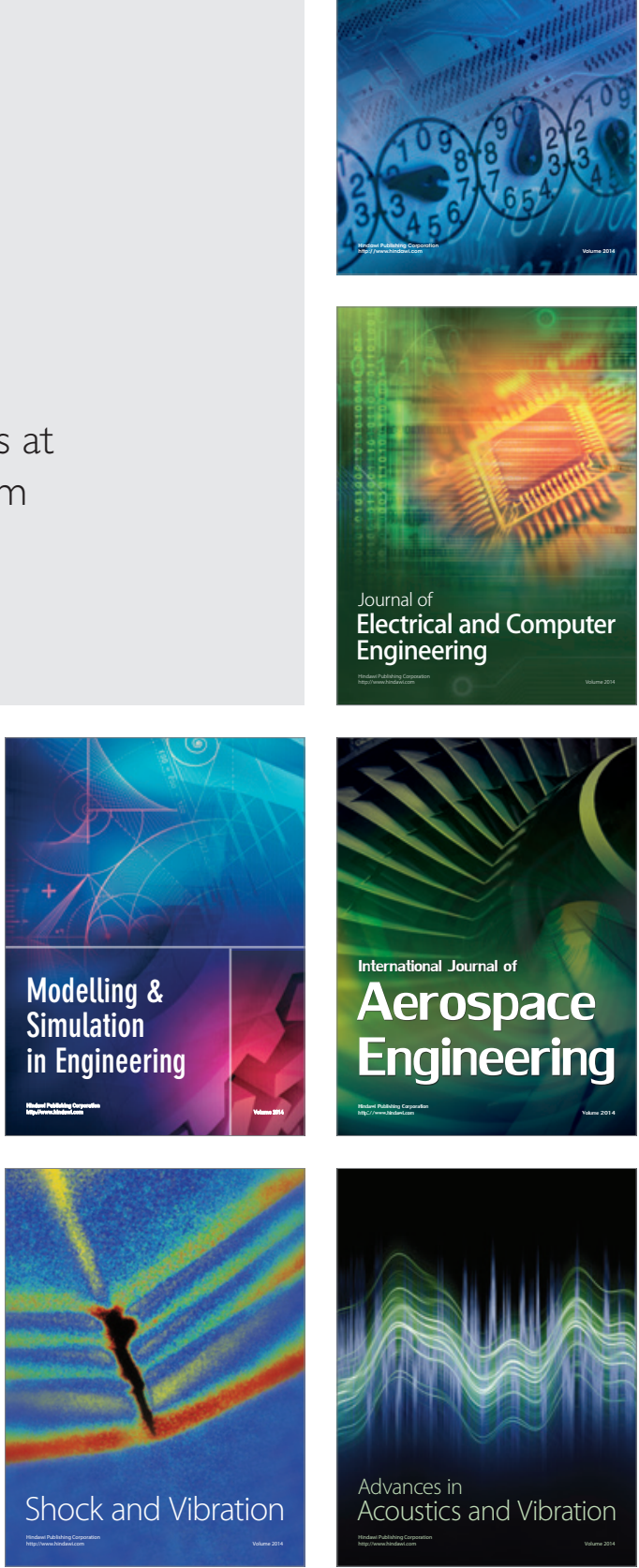